\title{
Commerce électronique et régulation des échanges internationaux
}

\author{
Philippe BARBET
}

\author{
Université de Paris 13 et CEPN-CNRS
}

\section{Introduction}

La principale particularité du commerce international est que la notion de frontière reste pertinente car les pays peuvent toujours protéger leurs offreurs domestiques derrière des barrières tarifaires (droits de douane) et non tarifaires (normes, restrictions quantitatives...). Depuis la fin de la seconde guerre mondiale, la tendance est à la réduction du protectionnisme mais les Etats-Nations gardent un rôle non négligeable dans la régulation des échanges internationaux au travers en particulier des politiques tarifaires, fiscales et commerciales.

Le développement du commerce électronique remet en question la notion de distance et en particulier le rôle des frontières. Il contribue, avec le développement des firmes transnationales et la croissance du pouvoir de L’Organisation Mondiale du Commerce (OMC) à accélérer le recul du rôle des Etats-Nations dans la régulation des échanges internationaux. Au delà, la diffusion internationale du commerce électronique perturbe le fonctionnement du système traditionnel de régulation des échanges et soulève de nouvelles questions.

Comme nous le montrerons dans un premier temps, l’importance des échanges électroniques internationaux est actuellement relativement faible mais son potentiel de croissance est important. En outre, l'existence de certains biens dits « numérisables » obligent à redéfinir les nomenclatures traditionnelles et en particulier la différence entre biens et services qui fonde les négociations commerciales internationales. Dans un second temps, nous analyserons les conséquences de l'effort de démantèlement des droits de douanes sur le commerce électronique international sur les recettes douanières et nous analyserons les principales conséquences de la création de ce « duty free cyberspace ». Enfin, nous montrerons que le 
commerce électronique oblige à une réflexion profonde sur les questions de fiscalité directe, indirecte et au-delà sur certains fondements de la relation d'échange.

\section{1 - L'importance du commerce électronique international et les enjeux de la classification des échanges entre biens et services}

Le commerce électronique est en phase de décollage et son importance dans l'ensemble des échanges internationaux reste faible. Toutefois, nous montrerons dans un premier temps que le potentiel de croissance est important et que ce phénomène ne restera sans doute pas marginal. Une grande partie des échanges de biens et de services sera peu affectée par la numérisation mais pour certains biens qui peuvent être vendus à la fois sous forme tangible et électronique, nous montrerons dans un second temps qu'il est nécessaire de conduire un indispensable effort d'analyse et de classification.

\section{1-1- $\quad$ Le commerce électronique international : situation actuelle et évolutions}

L’OMC définit le commerce électronique comme «la production, publicité, vente et distribution de produits par les réseaux de télécommunication » [OMC 1998] Pour l’Union Internationale des Télécommunications (ITU) a croissance forte de la diffusion de l'Internet avec plus de 141 millions d'ordinateurs connectés et près de 500 millions d'internautes en 2001(600 millions prévus en 2002) [ITU 2001] est largement liée à l’utilisation de plus en plus fréquente de ce réseau pour des transactions commerciales (figure 1). Ainsi, pour la Commission des nations Unies pour le Commerce et le Développement [CNUCED[2000] le commerce en ligne devrait représenter entre 10 et 25\% des flux d'échange internationaux en 2003.

Figure 1

L’évolution du commerce électronique (en millions de dollars courants) 


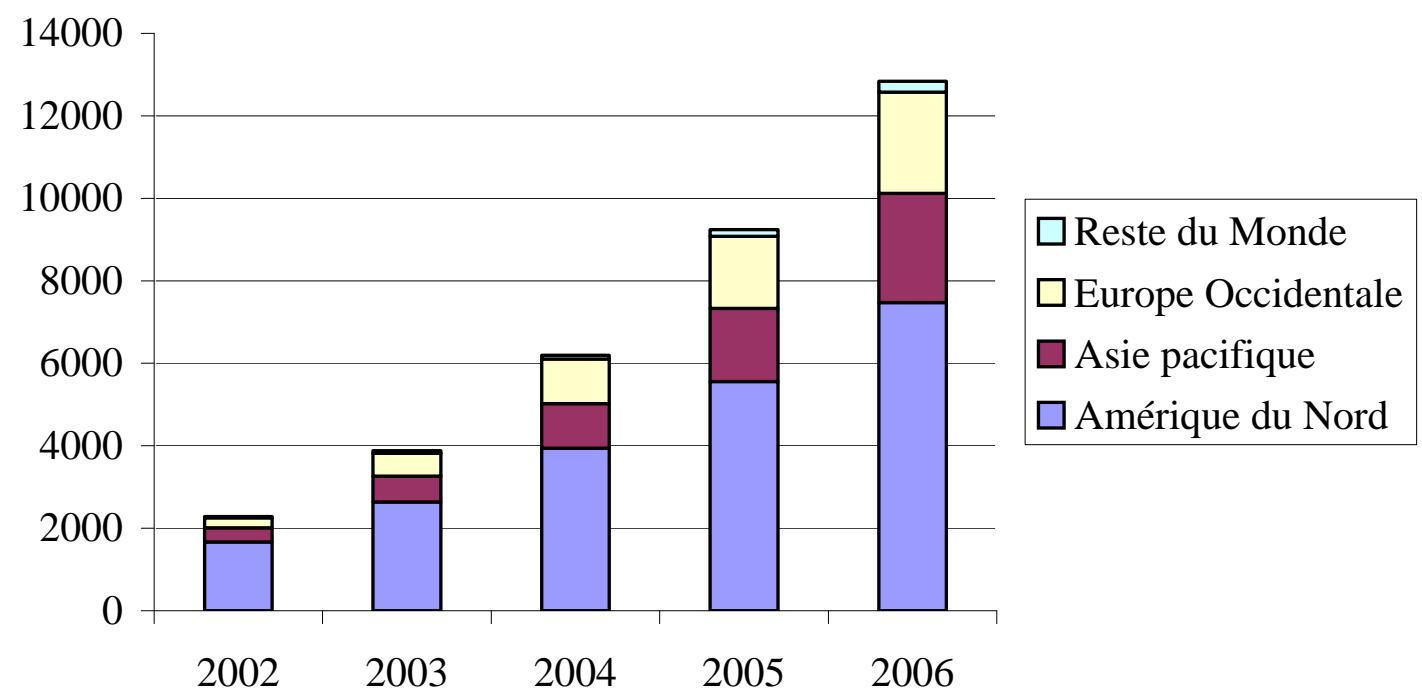

Sources: FORRESTER dans OMC [2002]

L'électronisation du commerce est un phénomène dans lequel on peut distinguer trois étapes principales [SCHUKNECHT 1999]. La première étape est celle de l'échange d'informations préalable à l'achat qui comprend notamment la publicité (flux d’information du vendeur vers l'acheteur) et la recherche d'information sur la qualité ou la localisation des produits (de l'acheteur vers le vendeur). La seconde étape est celle de l'acte d'achat qui comprend en particulier la commande et le paiement. La dernière est celle de la fourniture à l'acheteur du bien ou du service sur lequel porte la transaction. Toutes ces étapes peuvent inclure des flux d’échange électronique et ceux ci sont particulièrement fréquents dans les deux premières. La troisième étape est celle où, à priori, le potentiel d'échanges électroniques est le plus limité. Un certain nombre de services peuvent certes être fournis sous forme électronique (services financiers, conseil...) mais une grande partie des biens doivent faire l'objet d’une livraison physique. Pour ces biens, la notion de commerce électronique se réduit au deux premières étape et n’est donc pas très différente de la vente à distance.

Il existe toutefois un type de biens qu'il est particulièrement intéressant d'étudier dans le contexte du développement du commerce électronique. Il s’agit des biens « numérisables » : c'est à dire des biens qui peuvent être vendus sous forme traditionnelle (physique) mais aussi sous forme numérique. On parle de «biens numérisables" pour désigner les biens d'information comme les livres, les journaux, les logiciels ou certains produits de l'audiovisuel comme la musique et les films. 
Les échanges internationaux de biens numérisables ne représentent qu'environ $1 \%$ du commerce mondial mais, comme le montre la figure 2, les taux de croissances sont particulièrement importants pour des produits comme les jeux vidéo et les médias enregistrés. Le taux de croissance moyen est d'environ $10 \%$ par an soit 1,5 fois la croissance annuelle moyenne des échanges sur la période considérée.

Figure 2

Les exportations mondiales des principaux biens « numérisables »

(Millions de \$ et croissance)

\begin{tabular}{|c|c|c|}
\hline Types de biens & Millions de \$ & $\begin{array}{c}\text { Croissance annuelle moyenne } \\
\mathbf{1 9 9 0 - 1 9 9 6} \text { en \% }\end{array}$ \\
\hline Films & 375,1 & 4,2 \\
\hline Imprimés & 11099,2 & 7,6 \\
\hline Journaux & 4774,9 & 6,4 \\
\hline Publicité & 3864,9 & 5,6 \\
\hline Autres imprimés & 5138 & 9,6 \\
\hline Jeux vidéo & 2983,8 & 40 \\
\hline Bandes magnétiques enregistrées & 1718,6 & 1,1 \\
\hline Autres médias enregistrés & 13774,1 & 16,8 \\
\hline TOTAL & 43729 & 10,6 \\
\hline
\end{tabular}

Sources : MATOO \& SHUKNECHT [2000]

L'électronisation est essentielle pour l'internationalisation de certains services et est même souvent à la source du développement transfrontière de services autrefois strictement nationaux comme la santé. Les services juridiques peuvent être fournis par téléphone, les informations peuvent être envoyées par fax, les paiements se font électriquement. Enfin, les télécommunications sont elles-mêmes considérées comme une activité de service. Les flux électroniques transfrontière de services représentaient en 1995 environ 370 milliards de dollars, soit environ 30\% des flux totaux d'échanges de services et $6 \%$ de l'ensemble des échanges commerciaux. La figure 3 montre que ce sont les échanges électroniques de services entre entreprises qui sont les plus importants, ceci très loin devant les services financiers et d'assurance. On remarque enfin que les services numérisables aux particuliers, qui regroupent essentiellement des services liés aux biens culturels ou récréatifs, sont relativement marginaux. Il convient toutefois de noter que de futurs évolutions dans la libéralisation 
internationale des services (santé, éducation...) pourraient servir de base à un développement notable du commerce électronique vers les particuliers.

Figure 3

Les exportations internationales de services numérisables (en millions de \$)

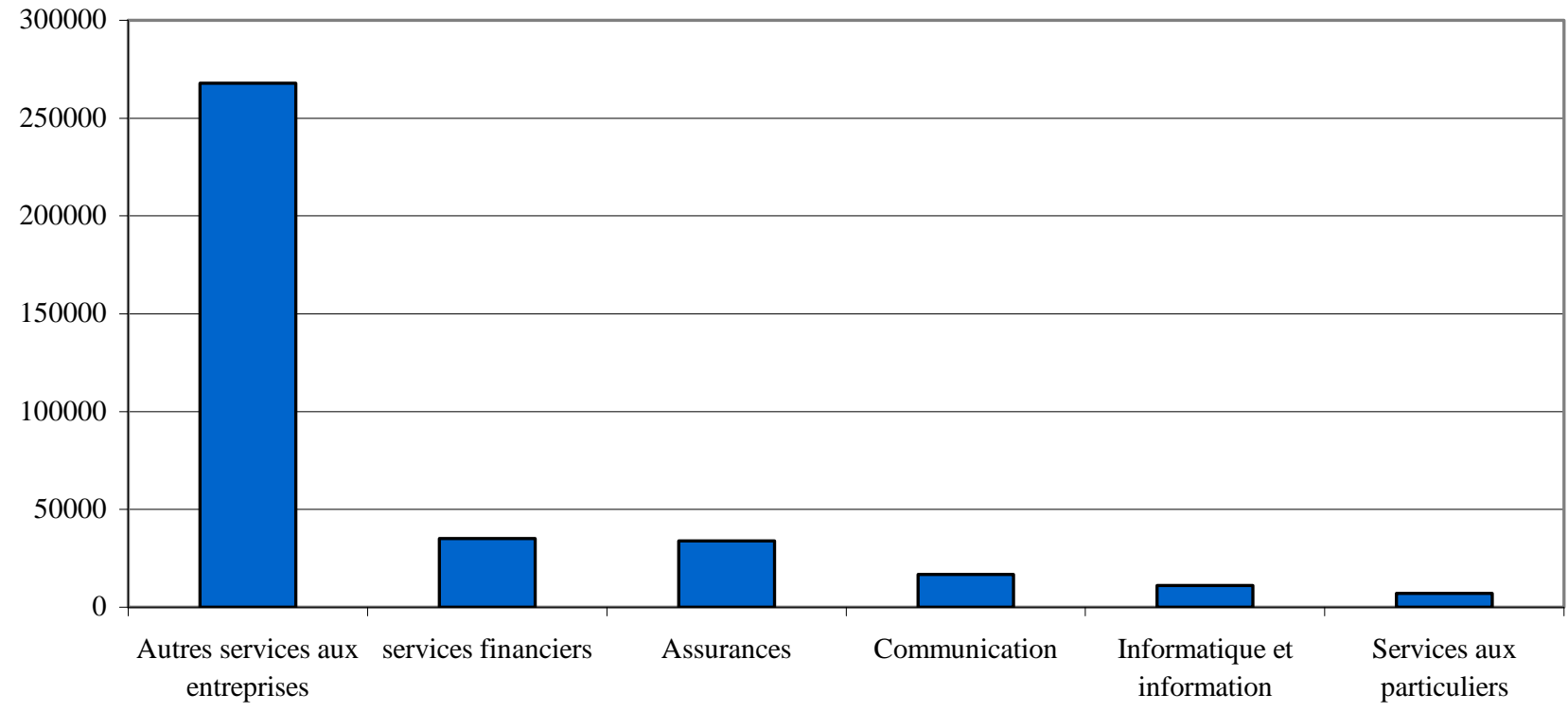

Sources : MATOO \& SHUKNECHT [2000]

Les flux d’échange internationaux de produits numérisables sont présentés dans la figure 4. On retrouve une configuration classique de l'économie contemporaine et en particulier de l'économie numérique caractérisée par une fracture entre les Pays Développés et les Pays en Développement. On constate en particulier que les principaux flux sont de type Nord -Nord ou Nord-Sud et que les flux Sud-Sud sont marginaux.

Figure 4

Les échanges internationaux de produits numérisables

(millions de \$)

\begin{tabular}{|c|c|c|c|c|c|c|}
\hline $\begin{array}{c}\text { Dest. } \\
\text { Origine }\end{array}$ & Afrique & $\begin{array}{c}\text { Amérique } \\
\text { latine }\end{array}$ & $\begin{array}{c}\text { Asie- } \\
\text { Océanie }\end{array}$ & $\begin{array}{c}\text { Pays en } \\
\text { transition }\end{array}$ & $\begin{array}{c}\text { Pays } \\
\text { développés }\end{array}$ & Monde \\
\hline Afrique & 4 & 1,1 & 37 & 0,1 & 198,9 & 242,3 \\
\hline Amérique & 0,5 & 468 & 88,4 & 0,8 & 2331,9 & 2907,9 \\
\hline
\end{tabular}




\begin{tabular}{|c|c|c|c|c|c|c|}
\hline Latine & & & & & & \\
\hline $\begin{array}{c}\text { Asie- } \\
\text { Océanie }\end{array}$ & 2,4 & 1,1 & 1359,7 & 388,9 & 2771,4 & 4581 \\
\hline $\begin{array}{c}\text { Pays en } \\
\text { Transition }\end{array}$ & 0,1 & 2,9 & 65 & 283,6 & 1528,4 & 1890,7 \\
\hline $\begin{array}{c}\text { Pays } \\
\text { développés }\end{array}$ & 21,7 & 401,3 & 2890,2 & 310 & 33670,2 & 37382,1 \\
\hline
\end{tabular}

Sources : CNUCED [2000] d'après la base de donnés COMTRADE des Nations Unies.

\section{1-2 La question de la différenciation et de classification entre biens et services.}

Le développement des échanges internationaux de biens numérisables n’est pas sans conséquences sur la manière dont sont abordées les questions de régulation des échanges internationaux. Les échanges de biens sont régis par les mécanismes du GATT repose sur les principes de non-discrimination (application de la clause de la nation la plus favorisée) et de la mise en place de concessions tarifaires permettant la réduction des droits de douanes. Pour les services, les accords GATS (Accord Général sur le Commerce de Services) précisent également que chaque Membre « accordera immédiatement et sans condition aux services et fournisseurs de services de tout autre Membre un traitement non moins favorable que celui qu'il accorde aux services similaires et fournisseurs de services similaires de tout autre pays ». Ce principe est toutefois limité par le fait que les pays peuvent maintenir des mesures incompatibles en proposant des exceptions.

La classification des biens numérisables en biens ou en services n’est donc pas neutre et il existe, sur ce point, une différence non négligeable entre la position américaine et européenne. Les Européens considèrent que toutes les transactions électroniques sont des échanges de services et relèvent donc des principes du GATS. Les Etats-Unis distinguent les services délivrés sur Internet qui doivent être couvert par le GATS des biens numérisables qui sont plus difficile à classer et préfèrent attendre les développements techniques futurs avant de les affecter dans les catégories de biens et de services.

L’approche européenne est donc potentiellement moins libérale car les accords du GATS ne libéralisent les marchés des services que pour les secteurs où les membres de l'OMC ont fait des propositions alors que les offres de libéralisation inscrites dans le GATT sont plus contraignantes. Pour sortir de ce conflit, un certain nombre de réflexions et de propositions émergent. La première consisterait à traiter les biens numérisables comme des services mais 
qu'ils soient automatiquement soumis aux règles de la clause de la nation la plus favorisée [MANN 2000]. On pourrait également envisager une solution qui éviterait les questions de classification et obligerait chaque membre de l'OMC à adopter la règle la plus libérale, que ce soit sous les principes GATS ou du GATT. Enfin, une proposition de l'Australie à l'organisation Mondiale du Commerce [OMC 2000] revient à considérer que les transactions de biens numérisables portent sur des échanges de droits de propriété intellectuelle (logiciels et musique en particulier). L'Australie note qu'il existe d'ailleurs un nombre croissant de transactions transfrontière concernant la propriété intellectuelle qui ne sont pas classées en biens ou en services (par exemple les droits de licence ou les royalties payés en contrepartie de l'utilisation de propriété intellectuelle). Ces transactions portant sur un droit de propriété intellectuelle peuvent se passer indépendamment de toute transaction qu'elle soit physique ou électronique. L'achat d'un CD musical ou d'un logiciel sur CD Rom ne porte pas légalement sur l'achat d'un support physique mais sur l'achat d'une licence (limitée) d'utilisation. Dans le cas d'un logiciel, la licence est d'ailleurs souvent étendue à la possibilité, pendant une certaine période, de télécharger des améliorations ou actualisations.

Les enjeux internationaux du commerce électronique sont actuellement émergents mais il est sans doute nécessaire de prendre conscience de l'ensemble des questions posées à l'architecture du commerce international. Nous montrerons dans le point suivant que la question des droits de douanes est sans doute une des plus intéressante.

\section{2 - Le commerce électronique international dans la libéralisation des échanges : les enjeux d'un « duty free cyberspace».}

Le commerce électronique, par ses caractéristiques, fait figure de laboratoire dans la libéralisation des échanges internationaux et dans la perte de contrôle des Etats de leurs possibilités de protection et de taxation des échanges. Nous verrons dans un premier temps que la pression des pays développés tend vers la mise en place et la permanence d'un espace totalement libéralisé pour le commerce électronique (duty free cyberspace). Toutefois, nous le montrerons dans un second temps, cette évolution n’est pas sans conséquences sur les recettes douanières et sur de possibles détournements d’échanges. .

2-1 L’Accord « Information Technology Assessment » et le « duty free cyberspace ». 
L’Information Technology Assessment (ITA) est un des trois arrangements postérieurs à l’Uruguay Round du GATT qui structurent la libéralisation du commerce électronique (avec l'accords GATS portant sur les services financiers et l'accord sur les télécommunications de base). Il est issu des travaux réalisés au sein de l'OMC et particulièrement d'une déclaration ministérielle sur le commerce des produits des technologies de l'information adoptée à la conférence de Singapour en décembre 1996. L’ITA est uniquement un mécanisme de réduction des droits de douane, il n’y a pas d'engagements contraignants pour les barrières non tarifaires aux échanges comme les quotas, normes ou achats publics protégés ceci même si la déclaration prévoie leur examen dans le futur. Les pays participants à l’ITA s’engagent en particulier sur les principes suivants :

- Tous les produits énumérés dans la déclaration doivent être couverts

- Pour tous ces produits, les droits de douane doivent être ramenés à zéro

Enfin, il est possible d'obtenir une prolongation des délais pour des produits sensibles mais pas d'exception

Les premières concessions tarifaires (baisse des droits de douane) prévues par l'ITA ont débuté à partir du $1^{\circ}$ juillet 1997 . Cet accord a permis l'élimination progressive des tarifs douaniers sur un ensemble important de produits de haute technologie comme les semiconducteurs, les ordinateurs, les équipements informatiques et les équipements de télécommunication. Il s'agit de l'unique accord pour lequel les participants se sont entendus sur une liste de produits pour lesquels tous les tarifs seront supprimés L’accord ITA doit être périodiquement modifié pour tenir compte en particulier des innovations technologiques et des différences dans les nomenclatures utilisées. Certains pays et en particulier les Etats-Unis [USTR 2000] souhaitent la mise en place d'un ITA II qui devrait inclure de nouveaux produits et de nouveaux pays comme la Chine après son accession à l'OMC. Le nouvel arrangement devrait également couvrir les barrières non tarifaires dans ce secteur et en particulier les normes et standards.

La proposition de constitution d'un « duty free cyberspace » est une initiative des EtatsUnis soumise à l'OMC en 1998 et qui encourage les Etats à ne pas appliquer de droits de douane sur les échanges électroniques internationaux. Il s’agit de mettre en place un moratoire temporaire sur les droits de douanes pour tous les produits livrés par Internet. La motivation affichée par les promoteurs de cette initiative est la difficulté de distinguer entre les biens tangibles et électroniques délivrés sur l’Internet et le brouillage de la distinction traditionnelle 
entre les biens et les services que nous avons mentionné dans la première partie. Cette proposition s'inscrit dans la pratique existante pour les communications téléphoniques et, malgré quelques difficultés dues à l'échec de la conférence de Seattle, devrait servir de base pour les échanges électroniques internationaux [LAPIERRE \& DONZEL 2000]

Le statut de ce moratoire temporaire est devenu incertain après l'échec de la conférence ministérielle de Seattle. Les Etats-Unis soutenaient que le moratoire est toujours en vigueur puisque la conférence de Seattle n'était pas terminée simplement suspendue. Pour les Européens, le moratoire allait jusqu’à Seattle et que la conférence soit finie ou suspendue, il n’y a pas d'accord automatique pour élargir le moratoire. La conférence ministérielle de Doha a décidé de prolonger le moratoire au moins jusqu’à la prochaine conférence ministérielle qui se tiendra en 2003 au Mexique.

\section{2-2 Les conséquences sur les recettes douanières et les risques de détournement d 'échanges.}

Le moratoire sur les droits de douane pour les échanges électroniques conduit mécaniquement à une réduction des recettes douanières des Etats. Toutefois, il convient de noter d'une part que les droits de douane ne constituent plus une recette budgétaire majeure dans la plupart des pays et d'autre part que, selon les estimations de l'OMC, l'impact du moratoire sur les recettes douanières est très faible sauf pour la Hongrie et pour la Chine comme le montre la figure 5

Figure 5

Recettes douanières des échanges des produits de médias « numérisables » en \% des recettes des Etats (1996)

\begin{tabular}{|l|c|c|c|}
\hline Pays & $\begin{array}{c}\text { Recettes } \\
\text { (millions de \$) }\end{array}$ & $\begin{array}{c}\text { \% des droits de } \\
\text { douane }\end{array}$ & $\begin{array}{c}\text { \% des recettes } \\
\text { publiques }\end{array}$ \\
\hline Pays développés & 233.4 & 0.7 & 0.01 \\
\hline $\begin{array}{l}\text { Pays en Développement } \\
\text { Dont : }\end{array}$ & 613.5 & 0.9 & 0.13 \\
\hline Argentine & 44.6 & 2.9 & 0.13 \\
\hline Brésil & 28.1 & 1.9 & 0.16 \\
\hline Chine & 107.3 & 11.6 & 0.95 \\
\hline Colombie & 12.9 & 1.4 & 0.11 \\
\hline Corée & 162.7 & 2.4 & 0.15 \\
\hline Hongrie & 7.7 & 14.9 & 0.07 \\
\hline
\end{tabular}




\begin{tabular}{|l|c|c|c|}
\hline Inde & 51.3 & 0.4 & 0.10 \\
\hline Maroc & 16.1 & 1.3 & 0.23 \\
\hline Mexique & 13.8 & 1 & 0.04 \\
\hline Philippines & 20.9 & 0.5 & 0.13 \\
\hline Thaïlande & 67.4 & 1.3 & 0.20 \\
\hline
\end{tabular}

Sources : GOOLSBEE 1998

En revanche, les conséquences de ce moratoire ne sont pas insignifiantes au regard de la théorie traditionnelle des échanges internationaux. Ainsi, pour MATTO \& SCHUKNECHT [2000], la non-perception des droits de douane pour un mode particulier de livraison (électronique) alors que les mêmes produits livrés traditionnellement sont soumis au tarif douanier conduit à la création mais aussi au détournements d’échange. Ces deux effets ont été analysés au début du siècle par Jacob Viner pour les accords douaniers préférentiels comme l’Union Douanière pour la construction européenne ou les accords de libre échange comme l'ALENA en Amérique du Nord. La suppression des droits de douane pour le commerce électronique conduit à une baisse des prix et donc une augmentation des échanges (effet de création). Il y a risque de détournement si, dans le cas du commerce électronique, le choix de ce mode de distribution est économiquement moins efficient et donc intéressant uniquement par l'avantage douanier qu'il procure. Pour le commerce électronique, le risque de détournement est sans doute peu important compte tenu de l'efficacité en général supérieure du mode de livraison électronique par rapport au mode physique ${ }^{1}$. Toutefois, la décision de l'OMC de ne pas imposer de droit de douane sur le commerce électronique crée une discrimination car des produits identiques sont taxés différemment selon le mode de livraison. En effet, de la musique ou un logiciel importés sous forme électronique sont exemptés mais taxés lorsqu'ils sont importés par des moyens classiques (quelques pays font payer une taxe sur le support physique et non pas sur le contenu). L'exemption des droits de douane pour les transactions électroniques est contradictoire avec le principe de neutralité technologique qui interdit toute discrimination dans le commerce selon son mode de livraison.

La question de la non-imposition de droits de douanes pour le commerce électronique s'inscrit dans une double dynamique. La première est celle, générale, de la réduction des barrières douanières qui a été engagée dès 1947 par le GATT et est poursuivie par l’OMC. La seconde est la volonté de la plupart des pays favoriser la croissance du commerce électronique en minimisant les coûts pour les consommateurs. Toutefois, et comme nous l'avons montré, la

\footnotetext{
${ }^{1}$ On peut supposer un que la connexion Internet est incluse dans un forfait et que l'ordinateur représente un investissement fixe. Dans ce cas, le coût marginal de la livraison électronique est nul
} 
question de la discrimination entre les droits de douane selon le mode de livraison nécessite encore un approfondissement.

\section{3 - La nécessaire évolution de la fiscalité indirecte et directe}

La question de l’imposition des transactions réalisées par le commerce électronique fait l'objet de nombreuses réflexions et travaux menés tant au niveau international que national. Au niveau international, l'OCDE a établi les principes d'une «bonne fiscalité » pour les échanges électroniques (fiscalité indirecte) et réalisé des avancées sur la définition des critères de détermination géographique d’une activité commerciale (fiscalité directe). Les principes de base de la fiscalité du commerce électronique ont été établis lors de la conférence ministérielle de l’OCDE à Ottawa en 1998. Il était nécessaire de réaliser un compromis entre les pays qui soutenaient le principe d'un développement sans contraintes du commerce électronique et ceux qui proposaient la création de nouveaux impôts [BUYDENS ]. L’OCDE a proposé que les principes généraux de la fiscalité s’appliquent au commerce électronique, que les transactions électroniques soient taxées comme des services et sur le lieu de leur consommation, elle a enfin rejeté les propositions de mise en place de taxes spécifiques comme la «taxe à l’octet». En février 2001, les TAG (technical Advisory Group) de l’OCDE ont précisé les principes nécessaire à la mise en place d’une fiscalité efficace du commerce électronique.

Les principes d'une « bonne » fiscalité du commerce électronique pour l’OCDE

Neutralité : La fiscalité doit être neutre et équitable entre les différentes formes de commerce électronique et avec le commerce traditionnel et notamment éviter la double imposition

Efficacité : Les coûts pour les entreprises et les administrations de la perception des impôts doivent être minimisés.

Clarté et simplicité : Les règles de la fiscalité doivent être claires et faciles à comprendre

Fiabilité et loyauté : La fiscalité doit permettre de produire le bon montant de recettes au bon moment, les possibilités d'évasion fiscale doivent être minimisées

Flexibilité : Le système d'imposition doit être flexible et dynamique pour permettre de prendre en compte les développements technologiques et commerciaux.

Source : J. OWENS, taxation in a wired world, OCDE 2000 
Dans le domaine de la fiscalité indirecte, il est souhaitable, mais sans doute à plus long terme comme nous le montrerons dans un premier temps, d'harmoniser les systèmes existants pour éviter les risques de frictions illustrés par le débat transatlantique sur les nouvelles modalités de collecte de la TVA en Europe pour les transactions électroniques. Concernant la fiscalité directe, nous montrerons dans un second temps que la question principale est celle de la définition des notions « d'établissement permanent » et de "pays d'origine », ce qui peut paraître paradoxal pour une activité dont la particularité est justement d'être relativement affranchie des contraintes géographiques.

\section{3-1 La fiscalité indirecte et les divergences entre l'Europe et les Etats-Unis}

Le développement du commerce électronique est relativement neutre pour la fiscalité indirecte pour tous les échanges qui impliquent la livraison finale d'un bien tangible. Si l'Internet est utilisé uniquement pour la recherche, la commande et le paiement de biens qui sont ensuite acheminés de manière traditionnelle, la situation est à priori la même que pour la vente à distance et il n'y a pas de spécificité sur ce point du commerce électronique. Lors de transactions internationales, la TVA et la taxe sur la vente de détail sont prélevée à la frontière avec les droits de douane et répercutée directement sur les prix de vente aux consommateurs. Les difficultés de taxation concernent essentiellement les ventes de produits numérisables puisque dans ce cas, il n’y a pas de franchissement physique de frontières. Pour ce type de biens, l’OCDE a proposé que ces transactions soient traités comme des échanges de services et non de biens, mais cette proposition place le commerce électronique dans une catégorie de transaction pour lesquelles les règles fiscales ne sont pas définies de manière précises.

La croissance de l'Internet a donc pour effet d'exacerber des difficultés qui préexistaient pour la taxation des services. Devant cette situation, La tentation est donc très forte de résoudre les difficultés en exonérant le commerce électronique de toute fiscalité nouvelle comme aux Etats-Unis avec l’Internet Tax Freedom Act de 1998

\section{Les principes de l'Internet Tax Freedom Act aux Etats-Unis}


L’Internet Tax Freedom Act (ITFA) est entré en vigueur le $1^{\circ}$ octobre 1998. il exempte les transactions électroniques réalisées aux Etats-Unis de toute nouvelle taxe pendant 3 ans. L’IFTA recommandait également de modifier les principes de la fiscalité indirecte aux EtatsUnis et confiait cette initiative à une commission (Commission Gilmore) composée de représentants de l'industrie, des consommateurs et du gouvernement. Cette commission n'a pas pu atteindre la majorité des 2/3 pour proposer une recommandation au Congrès américain. Toutefois, une majorité simple s'est dégagée pour proposer une prolongation de 5 ans du moratoire et une simplification du système de la fiscalité sur les ventes de détail aux EtatsUnis.

La commission a proposé que les produits numérisables ne soient pas imposés ainsi que leur équivalent physique pour respecter le principe de la neutralité technologique. Bien que la commission Gilmore ne soit pas compétente dans le domaine du commerce international, elle a demandé à l'administration américaine de soutenir la prorogation du moratoire sur les droits de douanes pour le commerce électronique au sein de l’OMC.

On peut considérer que l'impact du Commerce Electronique en terme de réduction de la base imposable est faible et devrait le rester pendant un certain temps. A court terme, l'absence de fiscalité peut donc générer des externalités positives favorisant le développement du commerce électronique, ceci d'autant plus que les acheteurs par Internet sont très sensibles aux prix dans leur décision d'achat [GOOLSBEE 2000]. Toutefois, à plus long terme, il faut tendre vers la mise en place d'un système d'imposition qui soit neutre pour les ventes réalisées par des canaux traditionnels et celles réalisées par l’Internet [BOSWORTH 2002].

Les enjeux de l'imposition indirecte du commerce électroniques sont différents pour le commerce avec les particuliers (B to C) et le commerce entre entreprises (B to B). Rappelons que la fiscalité indirecte repose en grande partie sur le système de la TVA dans la plupart des pays développés et notamment et Europe alors qu'elle passe par une imposition des ventes de détail aux Etats-Unis.

Le système de TVA qui existe dans de nombreux pays pèse sur l'ensemble ventes mais les entreprises récupèrent la TVA sur leur consommation intermédiaire. La plupart des pays appliquent la TVA sur les produits importés mais par sur les exportations. 
La taxe sur les ventes de détail comme aux Etats-Unis est appliquée uniquement lors de la vente finale. Elle s’applique en général sur l'ensemble des biens et sur quelques services comme les télécommunications et les services financiers. Cette taxe est variable en terme de base et de taux selon les Etats. Le développement du commerce électronique pourrait d'ailleurs donner l'occasion, comme nous l'avons vu avec la Commission Gilmore, de mettre en chantier une réforme de la fiscalité indirecte aux Etats-Unis.

Le débat sur la fiscalité indirecte et notamment la TVA sur le commerce électronique a été récemment réactivé par une initiative européenne. Une directive du 12 février 2002 devrait obliger les sites de commerce électronique situés en dehors du territoire des 15 Etats membres à appliquer la TVA sur leurs ventes aux consommateurs européens. Cette nouvelle réglementation vise à mettre fin à la concurrence déloyale qui existe entre les fournisseurs européens soumis à la TVA et les fournisseurs extra européens qui n’y sont pas assujettis. Cette nouvelle réglementation s'appliquerait pour les ventes de produits numériques (logiciels, jeux, audiovisuel...) vendus à des particuliers (B to C) et ne s’appliquerait pas au commerce entre entreprises (B to B). Cette directive doit être ratifiée par le Parlement Européen et devrait entrer en application de manière progressive. La directive obligerait les fournisseurs situés en dehors de l'Union Européenne à s'enregistrer auprès des services fiscaux dans l'un des pays membre afin de lui verser la TVA calculée selon les taux des pays de destination des biens. Ce pays d'accueil reverserait ensuite à chaque pays de destination la TVA qui lui revient.

Ce projet est fortement critiqué par les Etats-Unis qui rejettent toute forme de taxation du commerce électronique en considèrent que cette initiative constitue une barrière protectionniste aux échanges. Les autorités américaines soulignent également l'existence d'un risque de violation du principe de neutralité technologique dans le cas la TVA sur les échanges numériques est plus importante que sur leur équivalent physique ${ }^{2}$ (livres et journaux dont les taux sont fortement réduits par exemple). La directive fait également l'objet de réserves de la part de certains pays européens comme la Grande-Bretagne qui soulignent notamment les difficultés de sa mise en œuvre effective (séparation entre B to C et B to B,

\footnotetext{
${ }^{2}$ Les éditeurs allemands ont déposé une plainte pour discrimination envers le commerce électronique puisque le même contenu éditorial est soumis à une taxe réduite de $7 \%$ s’il est vendu sous forme physique (livre, journal) et de $16 \%$ si il est vendu sous forme électronique comme un disque compact.
} 
nécessité de localiser géographiquement les acheteurs...). Enfin, les entreprises ont généralement un avis très partagé sur cette question en reconnaissant d'une part la nécessité de règles équitables de concurrence mais en craignant d'autre part que la directive, en augmentant les prix, freine le décollage du commerce électronique.

\section{2-2 La fiscalité directe et les enjeux de la localisation des offreurs}

La perception de l'imposition directe (sur le revenu des agents) repose d'une part sur la possibilité pour les administrations fiscales de définir le territoire pertinent sur lequel doit s'effectuer la taxation et d'autre part sur la détermination de la fraction du revenu attribuable à cette présence [BOSWORTH 2002]. La notion « d'établissement stable » est donc au centre de la réflexion consistant à établir si une activité a une présence commerciale suffisante dans un pays pour justifier la taxation. Pour le commerce électronique, cette notion d'établissement stable est plus difficile à manipuler que pour des transactions classiques. Le comité des affaires fiscales de l'OCDE est parvenu en janvier 2001 à un consensus en distinguant d'une part l'équipement informatique (le serveur) et les informations et les logiciels qui sont stockés ou utilisés sur cet équipement afin de le faire fonctionner. Cette distinction assez traditionnelle entre matériel et logiciel conduit à considérer qu’un site web en lui-même ne peut pas constituer un établissement stable. L’OCDE a également précisé les règles permettant de définir la notion d'établissement permanent qui est repris par les organisations internationales [CNUCED 2001].

- Un site Internet en soi ne peut pas être un établissement permanent ;

- Un serveur accueilli par un fournisseur de services Internet ne peut pas être considéré comme un établissement commercial fixe si le fournisseur ne réalise pas de transactions par l'intermédiaire du serveur ;

- Un serveur peut être un établissement commercial fixe, c’est à dire permanent, s’il appartient à une entreprise qui fait des transactions par l'intermédiaire du serveur ;

- Les fournisseurs de services Internet ne peuvent pas être des établissements permanents des entreprises dont ils accueillent les sites.

Au-delà de la stricte imposition directe, la notion d'établissement permanent et la localisation géographique des offreurs dans le commerce électronique sont donc les principaux points de 
discussion en matière de fiscalité directe du commerce électronique. L'exemple européen est, à cet égard, particulièrement intéressant à étudier.

En l'absence d'une harmonisation totale de plus en plus difficile à mettre en œuvre (notamment compte tenu des probables élargissements) l’Union Européenne a choisi de combiner le principe du «pays d'origine » avec l'établissement de normes juridiques minimales communes [PIAZOLO 2001]. Les règles juridiques de la transaction électronique sont celles du pays du vendeur et, en cas de litige, l'acheteur peut porter plainte dans son pays d'origine mais ce sont toujours les règles du vendeur qui s'imposent. Toutefois, et dans le but de réduire les risques pour les consommateurs et aider au développement du commerce électronique, la Commission européenne a proposé la mise en place de normes communes dans des activités « complexes » comme l’assurance et les produits financiers.

Au-delà, et pour des raisons commerciales, des offreurs peuvent avoir intérêt à signaler aux consommateurs potentiels les lois commerciales applicables pour la transaction électronique si celles ci sont plutôt favorables aux demandeurs. Les consommateurs pourraient également, pour leurs recherches sur Internet, afficher des préférences concernant les juridictions, la protection du consommateur, la protection de la vire privée, les caractéristiques des contrats et les considérations fiscales [FORRESTER 2000]. Il semble nécessaire d'envisager une certaine normalisation concernant des règles juridiques portant sur les contrats, la protection des copyrights et des marques, les questions de pornographie et de racisme par exemple [SETI 2000].

\section{Conclusion}

Nous avons présenté les principaux enjeux du développement du commerce électronique dans le cadre de la régulation des échanges internationaux. Nous avons montré dans un premier temps que si les échanges électroniques internationaux restent marginaux, leur croissance annoncée nécessite un effort de réflexion dans la classification entre biens et services des « biens numérisables ». Le développement du commerce électronique s’effectue au sein d'un mouvement plus général de libéralisation des échanges et nous avons montré, dans un second temps, les enjeux du commerce électronique dans les négociations multilatérales. Enfin, et dans un troisième temps, nous avons abordé les questions de fiscalité indirecte et directe du commerce électronique. 
Bibliographie

P. BARBET \& M. RAINELLI M. [2002] la régulation internationale de la nouvelle économie, contribution au colloque de l'ADIS, Nouvelle économie : théories et évidence, à paraître en 2002.

C BARSHESKY [2000] The networked world initiative: trade policy enters a new era. Federal Communications Bar Association, October 232000

B. BOSWORTH [2002] Taxation and e-commerce, Issues in Internet governance, Tokyo Club Research Meeting, January 24 - 25, IFRI , Paris

MATTOO A. \& SCHUKNECHT L. [2000] Trade Policies for Electronic Commerce , Working Paper $n^{\circ}$ 2380, World Bank, June.

OMC [2002] E-Commerce Next Wave: Productivity and Innovation, communication de J. Meringer, Séminaire sur les effets du commerce électronique sur le revenu, http://www.wto.org/french/tratop f/devel f/sem05 f/sem05 prog f.htm

OCDE [2001] Understanding the digital divide, OCDE

OCDE [2001] Electronic commerce : a cluster approach to the negotiation of input services, working party of the trade committee, TD/TC/WP (2000) 33/final

OMC [2000] Conseil des aspects des droits de propriété intellectuelle qui touchent au commerce, Le programme de travail sur le commerce électronique, communication de l’Australie IP/C/W/233, 07 décembre 2000.

OMC [1999b] « Programme de travail sur le commerce électronique », Conseil du commerce des marchandises, G/C/W/158, 26 juillet.

OMC [1999c] Programme de travail sur le commerce électronique, Conseil du commerce des services, S/C/W/115, 16 juillet. 
OMC [1999d] Programme de travail sur le commerce électronique, Conseil des ADPIC, IP/C/W/18, 30 juillet.

OMC [1998] Electronic commerce and the role of WTO, Geneva

D. PIAZOLO [2001], E-Commerce and the multilateral framework, Wirtschaftpolitische, vol 48, Nr 6 pp 625-634

SCHUKNECHT L \& PEREZ-ESTEVE R. [1999] « A quantitative assessment of Electronic Commerce », WTO staff working paper ERAD-99-01

UNCTAD [2000], Building Confidence. Electronic Commerce and Development, New York.

Ludger SCHUKNECHT [1999] A quantitative assessment of Electronic Commerce, WTO staff working paper ERAD-99-01 